\title{
Le Parquet General Pres La Cour Constitutionnelle : Un Ornement De Trop?
}

\author{
Yannick Miteo Ngombo ${ }^{1}$
}

\section{Résumé}

Le présent article donne une lumière sur les compétences d'une institution d'un genre particulier rattachée à la Cour constitutionnelle de la République démocratique du Congo : le Parquet général. Après avoir mis en lumière les attributions de celui-ci, il a été démontré que cette institution n'est qu'une fioriture dans l'arsenal de justice congolais étant donné que ses attributions peuvent bien être exercées aussi bien par la Cour elle-même que par le corps des conseillers référendaires ainsi que par les autorités politiques et technocrates économistes, voire par le reste du peuple. Ainsi, l'auteur conclut en proposant sa suppression pure et simple ou, et pour faire dans la mesure, l'instauration d'un recours au service du Parquet général près le Conseil d'Etat ou la Cour de cassation selon qu'on se retrouve respectivement devant un contentieux portant sur les actes ou les personnes.

\begin{abstract}
This article sheds light on the powers of a particular institution attached to the constitutional Court of the Democratic Republic of Congo: the general prosecutor office. After highlighting the attributes, it demonstrates that this institution is only a decor in the Congolese arsenal of justice since its powers can be exercised by the Court itself, the body of referendum advisers, political authorities, technocrats, economists, and even by the rest of the people. Thus, this analysis proposes the suppression of the general prosecutor office, or establishing a recourse to the service of the prosecutor general office attached to the Council of state or the superior Court respectively about litigations involving acts or people.
\end{abstract}

\section{Introduction}

Institution d'origine privatiste, le Parquet général (PG) s'est invité dans la justice constitutionnelle à travers une pure invention du constituant congolais ${ }^{2}$. Aux origines en effet, le Procureur était respectivement le représentant du Roi (Prince ou Seigneur selon les cas)

1 Assistant à l'enseignement au sein de la Faculté de Droit de l'Université de Kinshasa, Administrateur civil de la République démocratique du Congo et Chercheur au Centre d'Etudes et de Règlement de Différends Internationaux en Afrique (CERDIA en acronyme). $\mathrm{N}^{\circ}$ de contact : +243 997001077 /+243825184889. Email : miteongombo@gmail.com.

2 BALINGENE KAHOMBO, «L'originalité de la Cour constitutionnelle congolaise : son organisation et ses compétences », in www.hamann-legal.de, consulté le 22/07/2018, p.4. 
puis il est devenu, au fil de luttes pour les droits humains, le gardien des intérêts de la société toute entière ${ }^{3}$. Ainsi, à ce jour, c'est en tant que représentant du pouvoir exécutif et non des intérêts du chef suprême et en tant que gardien des intérêts de la société que le Procureur agit ${ }^{4}$. Comme nous le verrons plus loin, dans l'exercice de ses fonctions, il est difficile que celui-ci se retrouve en position réelle d'indépendance vis-à-vis du gouvernement.

Dans l'exercice de ses fonctions, la Cour constitutionnelle (CC) se fait assister du Parquet général, du Corps de conseillers référendaires et du Greffe ${ }^{5}$. En effet, le législateur congolais innove en instituant, de manière surprenante, le PG près la $\mathrm{CC}$ de la République démocratique du Congo (RDC). Cette forme d'organisation de la CC ne vit ni n'existe dans aucun autre Etat de tradition Romano-germanique, note non moins sans intérêt Bolle ${ }^{6}$.

Un point mérite cependant d'être éclairci : pourquoi rajouter l'adjectif général pour qualifier le PG près la CC? En effet, on n'associe l'adjectif général à Parquet que lorsque celui-ci supervise, outre ses traditionnelles fonctions, tout aussi les travaux du Parquet directement inférieur à lui. C'est ainsi qu'avant l'institution du Parquet près le Tribunal de paix ${ }^{7}$ chapoté par le $1^{\text {er }}$ Substitut du Procureur, il n'y avait que le Parquet de la République comme Parquet le moins hiérarchisé, supervisé par un Parquet général près la Cour d'appel lui-même coiffé par un Parquet général près la Cour de Cassation. Dans le souci de lever tout équivoque, on rajoute aux Parquets leurs Tribunaux ou Cours d'attache. Ainsi, nous avons le Procureur général près la Cour d'appel, le Procureur général près la Cour de Cassation. L'adjectif « général » fait donc référence à une charge de supervision. On est général par rapport à des positions inférieures. Etant donné que la Cour constitutionnelle ne constitue pas un ordre de juridiction ${ }^{8}$ et que les membres du PG près la $\mathrm{CC}$ sont désignés parmi

3 André BUTEAU, «Les fonctions de poursuivant, de gardien de l'intérêt public, de représentant de l'État devant les tribunaux et de conseiller juridique exercées par le ministre de la Justice et Procureur général du Québec », in www.conferencedesjuristes.gouv.qc.ca/files/documents/21/84/lesfoncti onsdepoursuivantdegardiendelinteretpublic.pdf, consulté le 22/07/2018, pp.244 à 262 .

4 Ibid.

5 Articles 12, 19, 20 de la loi organique $\mathrm{n}^{\circ} 13 / 026$ du 15 octobre 2013 portant organisation et fonctionnement de la Cour Constitutionnelle, JORDC, 54 ${ }^{\mathrm{ème}}$ année, numéro spécial du 18 Octobre 2013.

6 Stéphane BOLLE, «Vers une Cour constitutionnelle à la congolaise », in, http://www.la-constitution -en-afrique.org/ consulté le 07 juin 2018.

7 Institué par l'article 65 de la loi organique $\mathrm{n}^{\circ} 13 / 011$-B du 11 avril 2013 portant organisation, fonctionnement et compétences des juridictions de l'ordre judiciaire, JORDC, $54^{\text {ème }}$ année, ${ }^{\circ}$ spécial du 11 avril 2013, 2013. Sous le Régime de la loi de 1982 sur l’Organisation et la Compétence judiciaire, il n'existait pas un Parquet près le Tribunal de paix.

8 Articles 149 à 157 de la Constitution de la RDC, JORDC, $47^{\text {ème }}$ année, $n^{\circ}$ spécial du 18 février 2006; v. aussi Félix VUNDUAWE te PEMAKO et Jean-Marie MBOKO D'JANDIMA, Droit constitutionnel du Congo, vol. II, Bruxelles, 2012, p.1040. Notons cependant que l'exposé des motifs de la même Constitution dispose malheureusement et contradictoirement que « ... les Cours et Tribunaux ont été éclatés en trois ordres juridictionnels:

- Les juridictions de l'ordre judiciaire placées sous le contrôle de la Cour de cassation;

- celles de l'ordre administratif coiffées par le Conseil d'Etat et 
les procureurs faisant carrière dans l'ordre administratif ou judiciaire ${ }^{9}$, l'adjectif général après procureur ne reste plus qu'une broutille vide de tout sens.

Dans le cadre de la présente étude, il sera donc question d'analyser le bien-fondé de l'institution d'un PG près la $\mathrm{CC}$ eu égard aux fonctions lui dévolues, à son rôle et à sa mission. Outre le criticisme juridique, nous ferons appel au droit comparé en soutènement de notre thèse.

\section{A. Mission du PG près la CC}

En liminaire de la présente réflexion, nous avons retracé sommairement la mission du Procureur, des origines à ce jour. Déjà, en tant que gardien de l'ordre public et des intérêts de la société, il est de bon aloi de le placer sous le doigt directeur du gouvernement ${ }^{10}$, exécutant des lois gardiennes de l'ordre public ${ }^{11}$. C'est dans cet esprit qu'est intervenue la révision de 2011, de l'article 149 de la Constitution. En effet, l'exposé des motifs de la loi de révision constitutionnelle de 2011 dispose en son point 4 que :

«... L'amendement introduit à cet article consiste en la suppression du Parquet dans l'énumération des titulaires du pouvoir judiciaire. Celui-ci est dévolu aux seuls cours et tribunaux. Cet amendement remet ainsi en harmonie l'article 149 avec les articles 150 et 151 qui proclament l'indépendance du seul magistrat du siège dans sa mission de dire le droit ainsi que son inamovibilité ».

Ainsi, le constituant s'est vite empressé de corriger cette erreur d'écriture commise lors de la rédaction de la Constitution du 18 février 2006 puisque seul le juge jouit d'une indépendance absolue parmi les acteurs ou membres de la magistrature. C'est pourquoi le droit à un juge indépendant et impartial est élevé au rang des droits non dérogeables aux côtés du droit à la vie, du principe de légalité des crimes, peines et procédures ${ }^{12}$, etc. La Cour euro-

- la Cour constitutionnelle ». Le malaise est évident dans cette formulation. En effet, après avoir affirmé de façon nette que les domaines judiciaire et administratif étaient des ordres, le constituant évite d'en faire autant s'agissant du domaine constitutionnel. Qui plus est, l'exposé des motifs qui n'a de valeur qu'en ce qu'elle reprend l'esprit du texte ne saurait prévaloir cependant sur une norme impérative reprise dans le corps du texte.

9 Article 13, loi organique portant organisation et fonctionnement de la CC, supra, note 5 .

10 Article 70 de la Loi organique n $13 / 011-\mathrm{B}$ du 11 avril 2013, Op.cit., dispose que « les officiers du ministère public sont placés sous l'autorité du ministre ayant la justice dans ses attributions. Celuici dispose d'un pouvoir d'injonction sur le Parquet...».

11 Par ordre public l'on entend la tranquillité, la salubrité et la sécurité publiques. Avec l'évolution des besoins de l'heure, à l'ordre public y est ajouté des nouvelles finalités. Ainsi, les notions de «bon ordre, esthétique publique, moralité publique, la dignité de la personne humaine et la protection contre soi-même ». Lire en ce sens Philippe FOILLARD, Manuel de droit administratif, $14^{\text {ème }}$ éd., 2009-2010, pp.290-291.

12 Articles 6 et 14 du Pacte international relatif aux droits civils et politiques, adopté et ouvert à la signature, à la ratification et à l'adhésion par l'Assemblée générale dans sa résolution $2200 \mathrm{~A}$ (XXI) du 16 décembre 1966, entrée en vigueur le 23 mars 1976. 
péenne de droits de l'homme abonde aussi dans ce sens lorsqu'elle considère, de façon constante et sans revirement, que le juge du parquet ne remplit pas les conditions nécessaires d'indépendance ${ }^{13}$. Il lui est reproché entre autres sa subordination au Gouvernement puisqu'étant à la solde de sa politique pénale et de justice, son mode de désignation, son amovibilité ${ }^{14}$, etc. La République démocratique du Congo, en procédant à cette révision constitutionnelle, a rejoint l'esprit de la Convention européenne de droits de l'homme alors que la France continue, à quelques exceptions près, de considérer le magistrat du Parquet comme autorité judiciaire ${ }^{15}$ dotée d'une relative indépendance ${ }^{16}$, contrairement à ses collègues, magistrats du siège.

L'une des conséquences logiques de ce qui précède est que les procureurs sont ainsi rarement soumis à la procédure de récusation ${ }^{17}$ faute d'intérêt de la part du justiciable. Dans le but d'enfoncer définitivement le clou sur cette question, il sied de noter que le seul magistrat qui détient le pouvoir sur l'état des personnes - le pouvoir d'arrestation du procureur est en effet encadré par le juge qui veille à ce que celui-ci ne l'exerce pas abusivement- est le juge, aussi dit magistrat du siège dont l'indépendance n'est plus sujette à caution ${ }^{18}$. C'est à ce titre que le constituant a jugé bon de réserver à lui seul l'indépendance du pouvoir judiciaire $^{19}$. Soumettre le PG près la CC aux mêmes conditions statutaires que le juge de la $\mathrm{Cc}^{20}$ serait donc absurde en ce sens que le $\mathrm{PG}$, fut-ce celui près de la $\mathrm{CC}$, n'a pas été repris

13 CEDH, 10 juillet 2008, Medvedyev et autres c. France.; CEDH, 23 novembre 2010, Moulin c. France. Lire aussi Jean-François RENUCCI, «Un séisme judiciaire : pour la Cour européenne des droits de l'Homme, les magistrats du parquet ne sont pas une autorité judiciaire », 2009, p. 600, in www.dalloz.fr, et Jean-François RENUCCI, « L'affaire Medvedyev devant la grande chambre : les « dits » et les « non-dits » d'un arrêt important», 2010, p. 1386, in www.dalloz.fr, cité par MarcAntoine GRANGER, Le parquet, une autorité judiciaire indépendante?, in www.droitconstitutionne 1.org/congrèsNancy/comN6/grangerT6.pdf\&sa, consulté le 10 juin 2018, p.1.

14 Lire en ce sens les arrêts CEDH, 28 juin 1984, Campbell et Fell c. Royaume-Uni, requête n ${ }^{\circ} 7819 / 77$ et 7878/77, A 80, § 78 .

15 Cons. const., décision n ${ }^{\circ}$ 93-326 DC, 11 août 1993; Loi modifiant la loi n 93-2 du 4 janvier 1993 portant réforme du code de procédure pénale, Rec. 217, RJC I-552, considérant n ${ }^{\circ} 5$. Cons. const., décision $\mathrm{n}^{\circ}$ 97-389 DC, 22 avril 1997; Loi portant diverses dispositions relatives à l'immigration, Rec. 45, considérant $\mathrm{n}^{\circ} 61$ et Cons. const., décision $\mathrm{n}^{\circ}$ 2010-80 QPC, 17 décembre 2010, M. Michel F., J.O. 19 décembre 2010, p. 22374, considérant n 11, cité par GRANGER, note 13, p.8.

16 GRANGER, note 13, p.13. Relative parce qu'ils subissent des interférences de la part du gouvernement quoi que tempérées par le principe selon lequel « la plume est serve, la parole libre ».

17 Parmi les grands principes régissant les parquets, nous avons l'irrécusabilité. Le PG est cependant récusable lorsqu'il n'intervient pas comme partie principale à un procès. Ce principe peut à notre sens s'appliquer mutatis mutandis au droit constitutionnel processuel.

18 Article 149 de la Constitution de la République démocratique du Congo (RDC) telle que modifiée par l'article 1er de la Loi n ${ }^{\circ} 11 / 002$ du 20 janvier 2011 portant révision de certains articles de la Constitution de la RDC, JORDC, 47ème Année, Numéro spécial du 18 février 2006.

19 Constitution de la RDC, note 18, article 150 al.1.

20 En effet, la loi organique portant organisation et fonctionnement de la CC dispose en son article 13 alinéa dernier que « Ils (les magistrats du parquet) sont soumis au statut des membres de la Cour ». Et l'article 22 de l'ordonnance ${ }^{\circ} 16 / 070$ du 22 aout 2016 portant dispositions relatives au statut 
comme faisant partie du pouvoir judiciaire ${ }^{21}$. Lui reconnaitre donc le même statut que le juge constitutionnel serait lui attribuer l'avantage de toute l'indépendance et la stabilité que seuls ces derniers jouissent de plein droit.

Sans entrer dans les détails et comme souligné préalablement, il sied de préciser aux lecteurs que nous sommes de ceux qui pensons que la révision de 2011 n'a aucunement violé l'article 220 dans son aspect réservé au pouvoir judiciaire 22 .

Rentrant dans le fond de la présente réflexion, il convient de relever que la première et seule allusion au PG près la CC est faite à l'article 152 de la Constitution. Celle-ci dispose que :

"Le Conseil supérieur de la magistrature est l'organe de gestion du pouvoir judiciaire. Le Conseil supérieur de la magistrature est composé de :

1. Président de la Cour constitutionnelle;

2. Procureur général près la Cour constitutionnelle;

3. Etc. »

Cette disposition nous révèle que le Procureur général près la $\mathrm{CC}$ est membre du Conseil Supérieur de la Magistrature, CSM en sigle, et qu'à ce titre, il participe à la gestion du pouvoir judiciaire ${ }^{23}$. Il faut ensuite se référer à la loi organique portant organisation et fonctionnement de la CC pour découvrir les missions confiées au PG près la CC. Placé sous la direction d'un Procureur général, le PG, eu égard de l'article 14 de la loi organique portant organisation et fonctionnement de la $\mathrm{CC}$, exerce les missions ci-après :

"En matière pénale, il recherche et constate les infractions relevant de la compétence de la Cour, soutient l'accusation et requiert les peines. Dans les autres matières de la compétence de la Cour, il émet des avis motivés. Il assiste à toutes les audiences de la Cour. Il peut y présenter des observations ».

particulier des membres de la Cour constitutionnelle astreint au PG l'obligation d'impartialité. Or, selon les éléments de son dossier, le PG doit soutenir à charge ou à décharge, en d'autres mots il doit prendre position. Seul le juge doit rester impartial puisque devant annoncer le verdict, après avoir écouté toutes les parties en ce compris le PG. C'est lui aussi qui décide de l'état des personnes.

21 Art 149 de la Constitution de la RDC, dispose que « Le pouvoir judiciaire est indépendant du pouvoir législatif et du pouvoir exécutif. Il est dévolu aux cours et tribunaux qui sont : la Cour constitutionnelle, la Cour de cassation, le Conseil d'Etat, la Haute Cour militaire ainsi que les cours et tribunaux civils et militaires....».

22 Lire avec intérêt Joseph KAZADI MPIANA, La révision constitutionnelle congolaise du 20 janvier 2011 : considérations critiques d'un citoyen (juriste), p. 12, in www.droitcongolais.info, consulté le 10 juin 2018.

23 Il élabore les propositions de nomination, de promotion et de révocation des magistrats. Il exerce le pouvoir disciplinaire sur les magistrats. Il donne ses avis en matière de recours en grâce. Article 152 de la Constitution de la RDC, note 18. 
A noter aussi que « le Procureur général poursuit l'exécution des arrêts " ${ }^{24}$. « Il se saisit aussi d'office dans les trente jours suivant la fin des fonctions ${ }^{25}$, faute de cette déclaration, en cas de déclaration frauduleuse ou de soupçon d'enrichissement sans cause, la Cour constitutionnelle ou la Cour de cassation est saisie selon le cas $»^{26}$. " A l'exception des traités et accords internationaux, le Procureur général saisit d'office la Cour pour inconstitutionnalité des actes visés à l'article $43^{27}$ de la présente Loi organique lorsqu'ils portent atteinte aux droits fondamentaux de la personne humaine ou aux libertés publiques $»^{28}$. « Il autorise la levée des pièces des dossiers instruits par le Parquet général $»^{29}$.

Il ressort donc de la lecture combinée de la Constitution et de la loi organique portant organisation et fonctionnement de la CC que les seules prétendues compétences réellement dévolues au PG sont celles de rechercher des potentielles infractions commises par le Chef de l'Etat, le Chef du Gouvernement ainsi que leurs complices et coauteurs s'il en existe. En matière constitutionnelle, électorale et dans les matières dites non-contentieuses, il émet des avis $^{30}$ et des observations ${ }^{31}$.

La mission du PG près la CC étant ainsi précisée, il importe de passer au crible de la critique le but ou la raison d'être du PG près la CC.

\section{B. Inexistence d'une ratio-base à l'existence du PG près la CC?}

A cet effet, nous allons démontrer à travers quatre axes que l'utilité de l'institution d'un PG près la $\mathrm{CC}$ est, et reste, introuvable. Le premier axe est lié à l'introuvabilité de la mission première du Parquet. Le deuxième axe est lié à l'existence d'un corps de conseillers référendaires et des juges constitutionnels eux-mêmes.Le troisième axe porte sur la large ouverture de la saisine de la $\mathrm{CC}$ et, le quatrième axe, repose sur la nécessité de rentabiliser la gestion de l'Etat.

Passons en revue ces différents axes afin d'apporter d'amples arguments à la thèse ici soutenue.

24 Loi organique portant organisation et fonctionnement de la CC, note 5, article94.

25 Du Président de la République ainsi que des membres du Gouvernement.

26 Constitution de la RDC, note18, article 99 al 5.

27 Il s'agit des Lois, des actes ayant force de Loi, des édits, des Règlements Intérieurs des Chambres parlementaires, du Congrès et des Institutions d'Appui à la Démocratie ainsi que des actes règlementaires des autorités administratives.

28 Loi organique portant organisation et fonctionnement de la CC, note 5, article 49.

29 Loi organique portant organisation et fonctionnement de la CC, note 5, article 15.

30 Emprunté du droit civil, l'avis est rendu par écrit dans un délai de huit jours suivant la communication du dossier au Procureur. Loi organique portant organisation et fonctionnement de la CC, note 5 , article 89.

31 Emprunté du droit civil, il y recourt pour émettre son point de vue sur le déroulement du procès ainsi que sur les incidents survenus. L'observation peut-être écrite ou verbale. 


\section{L’introuvable mission du Parquet général près la CC}

La fonction du Parquet près la $\mathrm{CC}$ ne nous est révélée qu'à travers ses compétences reprises dans la loi organique portant organisation et fonctionnement de la CC. Or, pour comprendre la fonction première d'un parquet, il faut non seulement questionner l'histoire mais aussi se renseigner sur les textes l'ayant préalablement institué, fut-ce aux côtés d'autres juridictions.

L'encre de la loi relative à l'organisation et la compétence des juridictions de l'ordre judiciaire nous révèle en ses articles 66, 67 et 68 que :

«Le MP surveille l'exécution des actes législatifs, des actes réglementaires et des décisions de justice. Il poursuit d'office cette exécution dans les dispositions qui concernent l'ordre public. Il a la surveillance de tous les officiers de la Police judiciaire, des officiers publics et des officiers ministériels, sauf des agents du greffe et de l'office des huissiers. Il veille au maintien de l'ordre dans les Cours et Tribunaux sans préjudice des pouvoirs du juge qui a la police d'audience... ».

"En matière répressive, le Ministère public recherche les infractions aux actes législatifs et réglementaires qui sont commises sur le territoire de la République. Il reçoit les plaintes et les dénonciations, accomplit tous les actes d'instruction et saisit les Cours et tribunaux».

"En matière de droit privé, le Ministère public intervient soit par voie d'avis soit par voie d'action. Il donne obligatoirement son avis dans les cas prévus par la loi..." "

Passons dès à présent au crible les fonctions du ministère public en l'analysant au regard de la mission confiée au PG près la CC.

De l'analyse des articles ci-haut repris, il appert que les fonctions traditionnelles du Parquet général varient selon qu'il s'agit de la matière pénale ou civile.

1. S'agissant de sa compétence pénale

Le PG a pour rôle de rechercher les infractions commises aux textes législatifs et réglementaires $^{32}$. Le PG près la CC détient également des compétences similaires mais celles-ci sont très limitées et liées.

a. Une compétence limitée

Le PG est ténu par une double limitation : personnelle et infractionnelle ${ }^{33}$.

32 Loi organique portant organisation et fonctionnement de la CC, note 5, article 66.

33 En ce sens, l'article 163 de la Constitution : « La Cour constitutionnelle est la juridiction pénale du Chef de l'Etat et du Premier ministre dans les cas et conditions prévus par la Constitution ». 
La limitation personnelle tient au fait que le PG près la $\mathrm{CC}$ a une compétence très limitée dans la recherche des infractions en ce sens qu'il ne se limite qu'à la personne du Chef de l'Etat et du chef du Gouvernement, ainsi qu'à leurs co-auteurs et complices en cas de collaboration dans la réalisation ou la tentation de l'infraction ${ }^{34}$.

La limitation infractionnelle, quant à elle, ne tient qu'aux infractions dont pourraient se rendre coupable les deux personnalités précitées investies du pouvoir public. La poursuite des autres infractions étant suspendue par l'immunité temporelle leur accordée ${ }^{35}$, sauf celles commises au cours ou à l'occasion de l'exercice de leurs fonctions officielles ${ }^{36}$. Les infractions dont question ici sont des infractions politiques ${ }^{37}$ de haute trahison ${ }^{38}$, d'outrage au Parlement ${ }^{39}$, d'atteinte à l'honneur ou à la probité ${ }^{40}$ ainsi que pour les délits d'initié ${ }^{41}$ et pour les autres infractions de droit commun commises dans l'exercice ou à l'occasion de l'exercice de leurs fonctions.

Comme ci-avant précisé, il s'agit des infractions politiques. Au nom du principe sacrosaint de la séparation des pouvoirs, il serait de bon aloi que le pouvoir judiciaire ne s'im-

34 L'infraction tentée est punissable au même titre que l'infraction commise. Article XX du Code pénal congolais, Décret du 30 janvier 1940 tel que modifié jusqu'au 31 décembre 2009 et ses dispositions complémentaires.

35 Constitution de la RDC, note 18, article 167.

36 Constitution de la RDC, note 18, article 164.

37 C'est-à-dire celles dont le but recherché est politique. Lire Raphael NYABIRUNGU MWENE SON-

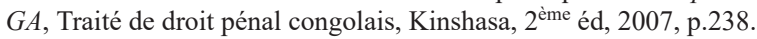

38 Il y a haute trahison lorsque le Président de la République a violé intentionnellement la Constitution ou lorsque lui ou le Premier ministre sont reconnus auteurs, coauteurs ou complices de violations graves et caractérisées des Droits de l'Homme, de cession d'une partie du territoire national.

39 Il y a outrage au Parlement lorsque sur des questions posées par l'une ou l'autre Chambre du Parlement sur l'activité gouvernementale, le Premier ministre ne fournit aucune réponse dans un délai de trente jours.

40 Il y a atteinte à l'honneur ou à la probité notamment lorsque le comportement personnel du Président de la République ou du Premier ministre est contraire aux bonnes mœurs ou qu'ils sont reconnus auteurs, co-auteurs ou complices de malversations, de corruption ou d'enrichissement illicite.

41 Il y a délit d'initié dans le chef du Président de la République ou du Premier ministre lorsqu'il effectue des opérations sur valeurs immobilières ou sur marchandises à l'égard desquelles il possède des informations privilégiées et dont il tire profit avant que ces informations soient connues du public. Le délit d'initié englobe l'achat ou la vente d'actions fondée sur des renseignements qui ne seraient jamais divulgués aux actionnaires. 
misce point dans la procédure de destitution comme c'est le cas en France ${ }^{42}$ et aux USA ${ }^{43}$ par exemple. En RDC, la décision du lancement des poursuites est certes prise par le Congrès mais celle de destituer éventuellement le président de la République appartient à la seule CC. Il se pose donc la question de la capacité du Président de la République à diriger l'Etat pendant qu'il fait l'objet de poursuites devant la CC. Serait-il en état de diriger ou passerait-il le gros de son temps à assurer sa défense? Le dernier cas nous parait être le plus probable. C'est ainsi que l'idéal serait de séparer la procédure judiciaire de celle de la destitution étant donné que le président est élu au suffrage universel et qu'à ce titre, il est redevable devant le peuple ${ }^{44}$ ou les représentants de celui-ci à travers lesquels il exerce sa souverainetét5 ${ }^{4}$ c'est-à-dire, les parlementaires. Ainsi, pour éviter au président de dissiper ses forces dans la bataille juridique et politique, la France et les USA ont opté pour une procédure de destitution avant toute poursuite judiciaire devant le juge naturel du désormais exchef d'Etat. Cette procédure a pour avantage certain de ne pas faire perdre au président de la République son droit le plus fondamental à faire appe ${ }^{46}$ des jugements rendus en première instance. Il serait donc temps que le constituant congolais revoie cette procédure afin de sauvegarder le droit d'appel du Chef de l'Etat, du Premier ministre ainsi que de leurs complice et coauteur.

Le PG est donc lié à veiller à ce que ces personnes ne se rendent pas coupables des infractions ci-haut énumérées. A noter aussi que toute personne peut soumettre au PG un cas de manquement dont se serait rendue coupable l'une de ces autorités. Au demeurant, ce dernier cas de figure est plus probable puisque seuls ceux qui côtoient les hautes sphères du pouvoir politique, ceux qui sont dans les rouages économiques et qui épient de près, par voie de conséquence, chaque acte de ces autorités qui sont à même de découvrir l'un ou l'autre manquement de leur part. Ainsi, pour le délit d'initié, les autorités de contrôle et de passation de marchés sont plus susceptibles de découvrir ce manquement. S'agissant de l'outrage au parlement, il va de soi que les parlementaires sont mieux indiqués dans la dé-

42 Article 68 alinéa premier de la constitution française telle que modifiée par la loi constitutionnelle n`2007-238 du 23 février 2007 portant modification du titre IX de la Constitution : « « Le Président de la République ne peut être destitué qu'en cas de manquement à ses devoirs manifestement incompatible avec l'exercice de son mandat. La destitution est prononcée par le Parlement constitué en Haute Cour ». Par Haute Cour ici, il s'agit du Parlement réuni en Congrès.

43 Maria Rosaria DONNARUMMA, Le statut juridictionnel du Chef de l'Etat et sa destitution, in www.nomos-leattualitaneldiritto.it/wp-content/uploads/2017/02/saggio-Donnarumma-Le-statut...corretto.pdf\&sa, pp.13-15, consulté le 11 juin 2018.

44 Idem, pp.4-5.

45 Il est patent de lire la section 2, du titre premier de la Constitution de la RDC du 18février 2006 intitulée « De la Souveraineté ». L'article 5 comprise dans cette section dispose que : « La souveraineté nationale appartient au peuple. Tout pouvoir émane du peuple qui l'exerce directement par voie de référendum ou d'élections et indirectement par ses représentants ».

46 Jacques Van COMPERNOLLE, Le double degré de juridiction et les exigences du procès équitable, in "Le double degré de juridiction. Etude de droit comparé », Sous dir., Jacques Van COMPERNOLLE, Achille SALETTI, Bruxelles, 2010, pp.3-14. 
couverte et l'établissement de ce manquement. L'atteinte à l'honneur et à la probité peut tout aussi être bien établie, sans être exhaustif, par tout procureur, par l'autorité de régulation bancaire, ainsi que par tous les rodés des hauts lieux d'affaires. Et il va sans dire que vu sa gravité, la haute trahison peut-être dénoncée ou portée à la connaissance des autorités compétentes pour analyse de l'opportunité de poursuivre par les médias et la clameur publique $^{47}$. Il y a donc lieu de se demander s'il faille instituer tout un parquet pour pareille compétence!

Non seulement que la compétence du PG est limitée en la matière, mais aussi faut-il préciser qu'elle est liée dans son exercice.

\section{b. Une compétence liée}

La poursuite du Président de la République est liée à la levée de l'immunité par le parlement réuni en congrès. A cet effet, l'article 168 de la Constitution dispose que "La décision de poursuites ainsi que la mise en accusation du Président de la République et du Premier ministre sont votées à la majorité des deux tiers des membres du Parlement composant le Congrès suivant la procédure prévue par le Règlement intérieur ».

Il ressort de cet article que, quand bien même le Procureur aurait réuni tous les éléments à charge, il devrait recevoir les libations du parlement, afin d'ouvrir les poursuites, par un vote renforcé qui, dans des Etats à faible démocratie et avec des majorités disciplinées ou mécaniques due au fait majoritaire, a très peu de chance d'aboutir pour être pesé dans nos dires. En plus, pour un magistrat qui est nommé par le Président de la République pour un mandat de 3 ans renouvelables ${ }^{48}$, ses chances de voir son mandat être renouvelé après une tentative de poursuite bloquée par parlement seront réduites en cendres. Le Procureur murira donc sa réflexion avant de s'aventurer dans des zones de turbulence du pouvoir réduisant ainsi sensiblement son efficacité. A quoi sert-il dans ce cas?

\section{S'agissant de sa compétence dans les matières autres que pénales}

En matière constitutionnelle, électorale et gracieuse, le PG près le CC émet des avis motivés et peut présenter des observations.

Rappelons qu'il est ténu d'assister à toutes les audiences de la Cour mais sans prendre part au délibéré.

47 Avec la floraison des mouvements citoyens, les violations constitutionnelles ont de plus en plus du mal à rester inaperçues. En plus, les violations graves de droits de l'homme violent profondément les consciences que la clameur publique suffirait à rependre l'information même dans des zones considérées comme fermées à l'information.

48 Loi organique portant organisation et fonctionnement de la CC, note 5 , article $13:$ «... Ils sont nommés, conformément au statut des magistrats, par le Président de la République, pour un mandat de trois ans renouvelable une seule fois, parmi les magistrats de l'ordre judiciaire ou administratif ayant au moins quinze ans d'expérience, sur proposition du Conseil Supérieur de la Magistrature ». 
Les avis motivés dont question ici ne sont rien d'autres que sa lecture fournie ainsi que sa position sur la question posée à la Cour. Ces avis doivent impérativement être écrits.

Les observations, quant à elles, sont ses remarques sur le déroulé du procès, bref sur les questions procédurales. Elles peuvent être écrites ou verbales.

C'est ici le lieu de jeter les jalons du débat abordé avec force et argument dans le point suivant. Est-ce nécessaire d'avoir une lecture de plus (pour certains) ${ }^{49}$ ou de trop (à notre avis) sur des questions purement constitutionnelles et donc d'une éprouvante technicité devant une Cour composée essentiellement des juristes rodés non seulement par leur domaine de compétence mais aussi par l'onction du dieu Chronos? Le point suivant nous renseignera davantage.

\section{L'existence d'un corps de conseillers référendaires et des juges eux-mêmes}

Nous émettrons notre lecture sur le profil des juges à la Cour avant de chuter sur celui des conseillers référendaires.

\section{Le profil des juges comme base suffisante}

La Constitution dispose en son article 158 que :

« La Cour constitutionnelle comprend neuf membres nommés par le Président de la République dont trois sur sa propre initiative, trois désignés par le Parlement réuni en Congrès et trois désignés par le Conseil supérieur de la magistrature. Les deux tiers des membres de la Cour Constitutionnelle doivent être des juristes provenant de la magistrature, du barreau ou de l'enseignement universitaire. Le mandat des membres de la Cour constitutionnelle est de neuf ans non renouvelable. La Cour constitutionnelle est renouvelée par tiers tous les trois ans. Toutefois, lors de chaque renouvellement, il sera procédé au tirage au sort d'un membre par groupe...».

Il ressort de cette disposition des observations intéressantes que nous recouperons de façon quantitative et qualitative.

Quantitativement, le nombre des juges composant la Cour constitue en soi un des gages d'un verdict expurgé de toutes tares juridiques. Déjà, les Saintes écritures nous révèlent que le salut réside dans le grand nombre de conseillers ${ }^{50}$. La Cour constitutionnelle ne siège valablement qu'avec le concours de 7 juges, pas moins, fut-ce pour des raisons d'urgence. Il est une pratique abondante à travers le monde que les Hautes juridictions soient composées d'une multitude de juges afin d'avoir plusieurs analyses et vider ainsi les éventuelles

49 Guy-Prosper DJUMA, Parquet général près la Cour constitutionnelle de la République démocratique du Congo, annuaire congolais de justice constitutionnelle, doctrine-jurisprudence, textes juridiques, vol.1, 2016, pp 89-110.

50 Livre de proverbes (La sainte bible, version Louis Second, 2006), Chapitre 11, verset 14. « Quand la prudence fait défaut, le peuple tombe; et le salut est dans le grand nombre des conseillers ».. 
contradictions des jugements précédents ou donner un arrêt qui aura les gages nécessaires d'être juridiquement exempt d'erreurs.

Qualitativement, il sied de noter que les juges de la Cour constitutionnelle doivent être dotés d'une onction de connaissances acquises au prix des années de travail dévouées aussi bien à la science juridique que dans des domaines connexes au droit. C'est ainsi que non seulement le deux-tiers des membres de la Cour doive être des juristes provenant de la magistrature, du barreau ou de l'enseignement supérieur mais ils doivent aussi disposer d'une expérience, a minima, avérée de quinze ans ${ }^{51}$ et cela sous peine de nullité de leur nomina$\operatorname{tion}^{52}$. Le tiers restant doit provenir du domaine politique ${ }^{53}$ et disposer aussi d'une expérience similaire aux juristes.

A ne pas négliger dans l'appréciation de l'exécution de la tâche qui est la leur de prendre en compte la durée de leur mandat ${ }^{54}$ qui est de neuf ans et du fait que celui-ci soit, au demeurant, non renouvelable. Cette disposition met le juge à l'abri d'une quelconque crainte de non renouvellement de son mandat par l'autorité nommant et assure, par sa durée, la confortabilité de son mandat. Comment ne pas assurer valablement son devoir d'ingratitude dans ce cas?

De même, les faits que le renouvellement triennal de la Cour se fasse par tirage au sort et que le Président de la Cour soit élu par ses pairs en sont des gages supplémentaires d'indépendance. Le tirage au sort est considéré par certains comme la meilleure expression de la démocratie et du suffrage en ce qu'il ne prend en compte ni la condition de l'individu, ni ses prouesses mais s'en remet exclusivement au tout puissant pouvoir de l'aléa ${ }^{55}$.

Il faut ajouter aussi le fait qu'il ne peut y avoir deux membres parents ou alliés jusqu'au troisième degré inclusivement ni plus d'un membre issu d'une même province ${ }^{56}$. Cette disposition vise à éviter que certains juges, poussés par des élans tribaux, puissent coaliser afin

51 Constitution de la RDC, note 18, article 159.

52 Loi organique portant organisation et fonctionnement de la $\mathrm{Cc}$, note 5 , article 4.

53 Par domaine politique ici, il faudrait entendre l'occupation des postes ou emplois dans des institutions politiques du pays à l'instar du gouvernement, du parlement et des institutions à caractère purement politique comme le Mécanisme de suivi de l'accord, le Conseil National de Suivi de l'Accord, etc. Le fait d'occuper un emploi dans un parti politique par exemple ne devrait pas être pris en compte.

54 En ce sens, Marcelin NGUELE ABADA, L'indépendance des juridictions constitutionnelles dans le constitutionnalisme des Etats francophones post-guerre froide : l'exemple du Conseil constitutionnel camerounais » in www.droitconstitutionnel.org, consulté le 09/06/2018, p.4.

55 Yves SINTOMER, Le pouvoir au peuple. Jurys citoyens, tirage au sort et démocratie participative, Extraits, in www.etienne.chouard.free.fr, 2007, pp.1-8, consulté le 18 juin 2018. Le tirage au sort a pour avantage d'écarter le choix des personnes sur la base des conditions liées à leur situation. Elle met tous les candidats sur le même pied et leur donne une égalité de chance.

56 Loi organique portant organisation et fonctionnement de la CC, note 5, article 2. 
de faire primer les intérêts du terroir au détriment de ceux de l'Etat. Assermentés ${ }^{57}$ de surcroît, les juges qui se rendraient coupables d'un quelconque manquement révélant leurs inféodations se rendront coupables de parjure.

De ce qui précède, il y a lieu de se demander comment les juges nommés dans pareilles conditions n'exerceraient-ils pas mieux la tâche qui est la leur au point de recourir à un avis, un réquisitoire ou une observation d'un Procureur dont les compétences avérées dans le domaine constitutionnel sont sujettes à débat? A moins qu'il ne soit un surhomme sorti tout droit de la théorie nietzschéenne. Sans oublier que la loi organique donne encore la possibilité au PG près la CC de recourir aux services du PG près la Cour de cassation ${ }^{58}$. N'était-ce pas mieux de rendre, au besoin, principal ce recours accessoire? On aurait ainsi une Cour qui siégerait, ne serait-ce que pour le besoin de la forme, avec le concours du ministère public.

Certains accuseraient le fait majoritaire de compromettre l'indépendance de ces juges en ce sens que la majorité au pouvoir s'assure de la désignation de six membres qu'elle apprécie à son goût. Mais, entre des juges jouissant de toutes les garanties d'indépendance et un Procureur nommé pour trois ans renouvelables et qui, de ce fait, et eu égard aux arguments ci-précédemment avancés, a quelque peu les mains et poignées liées?

Dans le but de mieux accomplir leurs fonctions, des conseillers référendaires sont affectés au service des juges.

\section{Le profil des conseillers référendaires comme base suffisante}

Sous inspiration belge ${ }^{59}$, il est institué un corps de conseillers référendaires à la CC. Leur nombre ne peut aller au-delà de 60. Ils sont placés sous l'autorité du Président de la Cour ${ }^{60}$. Recrutés sur concours, trois quarts d'entre eux doivent être juristes ${ }^{61}$. Ils doivent être impérativement de nationalité congolaise, titulaire d'au moins un diplôme de licence en droit ou tout autre diplôme équivalent, être de bonne moralité et avoir une expérience profession-

57 Loi organique portant organisation et fonctionnement de la CC, note 5, article 10 : [Avant d'entrer en fonction, les membres de la Cour sont présentés à la Nation, devant le Président de la République, l'Assemblée Nationale, le Sénat et le Conseil Supérieur de la Magistrature représenté par son Bureau. Ils prêtent devant le Président de la République le serment suivant : « Moi, ..., Je jure solennellement de remplir loyalement et fidèlement les fonctions de membre de la Cour Constitutionnelle de la République Démocratique du Congo, de les exercer en toute impartialité, dans le respect de la Constitution, de garder le secret des délibérations et des votes, de ne prendre aucune position publique, de ne donner aucune consultation à titre privé sur les questions relevant de la compétence de la Cour Constitutionnelle et de n'entreprendre aucune activité mettant en cause l'indépendance, l'impartialité et la dignité de la Cour ». Le Président de la République leur en donne acte].

58 Loi organique portant organisation et fonctionnement de la CC, note 5, article 18.

59 Articles 35-39 de la Loi spéciale belge sur la Cour constitutionnelle, 6 janvier 1989. En Belgique ils sont au nombre de dix-huit dont neuf d'expression française et neuf d'expression néerlandaise.

60 Loi organique portant organisation et fonctionnement de la CC, note 5, article 20.

61 Loi organique portant organisation et fonctionnement de la CC, note 5 , article 22. 
nelle d'au moins dix ans dans le domaine administratif, politique ou juridique. A noter que pour les Conseillers référendaires non juristes, il leur est requis une expérience de cinq années supplémentaires que les juristes ${ }^{62}$.

C'est ici l'occasion de se demander pourquoi imposer autant d'années d'expérience professionnelle alors qu'ils sont loin d'exercer les mêmes fonctions que les juges ou les Procureurs à la $\mathrm{Cc}$ ? La réponse à cette question nous est très vite donnée à travers les attributions de ce corps. En effet, les conseillers référendaires ont pour but d'assister la Cour dans l'étude et la préparation technique des dossiers dont elle est saisie ${ }^{63}$. Il appert donc que ceux-ci préparent les dossiers des affaires soumises à la Cour en dégageant les problèmes juridiques et proposant des solutions (voire des jugements pré-écrits) motivées en droit afin que le juge les agréent et analysent ceux-ci lors de leurs délibérés. Une expérience avérée s'impose sans nul doute pour l'accomplissement de pareille tâche. Toutefois, techniquement, il serait incommode de demander à un rodé des affaires juridiques voire constitutionnelles ayant autant d'années d'expérience à son actif de venir exercer les fonctions d'aide auprès des juges constitutionnels avec qui ils disposent des compétences quasi-similaires pour ne pas dire les mêmes. Revoir ce délai à cinq ans, par exemple, serait axiologiquement fondé puisque ceux-ci seront plus motivés à apprendre et travailler dur pour se voir devenir un jour juge constitutionnel à leur tour.

Eu égard de ce qui précède, nous sommes en droit de nous interroger sur le bien-fondé de l'avis ou de l'observation d'un Procureur dans le traitement d'un dossier passé au crible respectivement par les hautes compétences des conseillers référendaires, des membres du cabinet des juges puis des juges eux-mêmes? Sans compter que la CC a en outre la latitude de recourir, s'il échet, à l'expertise tant nationale qu'internationale ${ }^{64}$ ?

La large ouverture de la saisine de la CC contribue aussi à dévaloriser l'existence d'un $\mathrm{PG}$ près la $\mathrm{CC}$.

\section{La large ouverture de la saisine de la CC}

Il serait tentant de penser que le PG près la $\mathrm{CC}$ aurait des pouvoirs spécifiques et exclusifs de saisine de la Cour dans certaines matières relevant de sa compétence. Or, comme nous l'avons vu précédemment, même en matière pénale, il n'est pas l'autorité la mieux indiquée pour prendre connaissance et recenser les infractions relevant de la Cour.

Une lecture survolée de la loi pourrait cependant entretenir le flou sur des domaines réservés à la seule saisine de la Cour par le PG. En effet, l'article 49 de la loi organique portant organisation et fonctionnement de la Cour dispose qu'

62 Loi organique portant organisation et fonctionnement de la CC, note 5, article 20.

63 Loi organique portant organisation et fonctionnement de la CC, note 5, article 21 .

64 Loi organique portant organisation et fonctionnement de la CC, note 5, article 26. 
"à l'exception des traités et accords internationaux, le Procureur Général saisit d'office la Cour pour inconstitutionnalité des actes visés à l'article $43^{65}$ de la présente Loi organique lorsqu'ils portent atteinte aux droits fondamentaux de la personne humaine ou aux libertés publiques ${ }^{66}$.

Symptomatique ou hérétique, l'écriture de cet article vient directement après celle de l'article 48 de la même loi qui dispose que «toute personne peut saisir la Cour pour inconstitutionnalité de tout acte visé à l'article 43 de la présente Loi organique à l'exception des traités et accords internationaux ». A l'article 162 alinéas 2 et 3 de la Constitution de renchérir en affirmant que

" toute personne peut saisir la Cour constitutionnelle pour inconstitutionnalité de tout acte législatif ou réglementaire. Elle peut en outre, saisir la Cour constitutionnelle par la procédure de l'exception de l'inconstitutionnalité invoquée dans une affaire qui la concerne devant une juridiction ».

De ces articles, il ressort que le PG tout en ayant certes l'obligation de saisir d'office la Cc en cas de violation des droits fondamentaux contenue dans les actes infra-constitutionnels, accords internationaux exceptés, n'a cependant pas compétence exclusive en la matière puisque partageant ce pouvoir avec l'ensemble de la population congolaise. Dans la même veine, il est évident que le destinataire premier de ces normes aura plus d'intérêt à saisir la $\mathrm{CC}$ (puisque y assurant une veille juridique) que le PG qui, serait, dans ce cas de figure, surmené sans nul doute par l'examen, article par article, de chaque loi, acte ayant force de loi, édit, règlements Intérieurs des chambres parlementaires, du Congrès et des Institutions d'Appui à la Démocratie ainsi que des actes règlementaires des autorités administratives. En matière de droits humains, nous relevons que les ONGs de droits de l'homme qui foisonnent davantage à travers le pays font davantage une veille juridique afin d'expurger de tout texte les potentielles violations de droits humains.

Quoique l'action en inconstitutionnalité soit limitée dans un délai de six mois pour les lois et règlements et de 60 jours pour les traités et ce, suivant leur publication au journal officiel, le recours en inconstitutionnalité par voie d'exception reste cependant permanent et immanent en faveur de tout individu ${ }^{67}$.

Pour clouer définitivement au pilori cette institution creuse de sens, il sied de relever qu'une catégorie d'actes subisse un contrôle imposé de constitutionnalité avant leur entrée en vigueur limitant ainsi davantage les pouvoirs du PG. C'est le cas des lois organiques ${ }^{68}$,

65 (Il s'agit des Lois, des actes ayant force de Loi, des édits, des Règlements Intérieurs des Chambres parlementaires, du Congrès et des Institutions d'Appui à la Démocratie ainsi que des actes règlementaires des autorités administratives.).

66 Loi organique portant organisation et fonctionnement de la CC, note 5, article 49.

67 Constitution de la RDC, note 18, article 162 alinéa 3; Loi organique portant organisation et fonctionnement de la CC, note 5 , article 52 .

68 Loi organique portant organisation et fonctionnement de la CC, note 5 , article 44 . 
des règlements intérieurs des chambres parlementaires, du Congrès, des Institutions d'appui à la démocratie ainsi que leurs modifications ${ }^{69}$, les ordonnances prises après délibération en Conseil des Ministres par le Président de la République, en cas d'état d'urgence ou de siège ${ }^{70}$ et même des lois à promulguer sur saisine du Président de la République dans les dix jours qui suivent la transmission de la loi à son bureau, des présidents de chambres parlementaires ou encore par le dixième des députés ou sénateurs dans les quinze jours qui suivent l'adoption définitive de la loi $^{71}$. Dans ce cas de figure, la saisine se fera donc soit par le Président de la République, le président de chaque chambre parlementaire, le dixième des députés ou sénateurs ou encore par le président d'une institution d'appui à la démocratie.

A noter aussi qu'en matière d'interprétation des textes constitutionnels, la compétence lui (PG) échappe totalement puisque la loi organique et la Constitution ne confèrent ce pouvoir qu'aux seuls Président de la République, au chef du gouvernement, aux Présidents du Sénat et de l'Assemblée nationale, à un dixième des membres de chacune des Chambres parlementaires, aux Gouverneurs de province et aux Présidents des Assemblées provinciales.

En matière de conflit de compétence, la Cour est saisie par les présidents des chambres parlementaires ou par le chef du gouvernement en cas de conflit entre pouvoirs législatif et exécutif $^{72}$, sur saisine du chef du gouvernement, des présidents des assemblées parlementaires, des gouverneurs ou des présidents des assemblées provinciales selon les cas, en cas de conflit de compétence entre Etats et provinces $^{73}$ et par les parties au procès au cours duquel les juridictions judiciaire et administrative se sont reconnues compétentes en cas de conflit entre ordres de juridiction ${ }^{74}$.

En matière électorale, il va sans dire que seules les parties qui ont un intérêt particulier qui sont invités à saisir la Cour.

En cas d'absence de déclaration patrimoniale du président de la République ou de tout membre du Gouvernement, la Cour est saisie par un dixième au moins des députés ou des sénateurs pour constater la démission d'office du président de la République ou du membre du Gouvernement concerné ${ }^{75}$. Et dans les trente jours suivant la fin des fonctions, faute de cette déclaration ou en cas de déclaration frauduleuse ou de soupçon d'enrichissement sans cause, dans le chef d'une précitée autorité, la Cour sera saisie aux fins des poursuites pénales, à la requête du Procureur général agissant d'office ou sur plainte de toute personne intéressée, dans les trente jours suivant la fin des fonctions du président de la République

69 Loi organique portant organisation et fonctionnement de la CC, note 5 , article 45 .

70 Loi organique portant organisation et fonctionnement de la CC, note 5, article 46.

71 Loi organique portant organisation et fonctionnement de la CC, note 5, article 47.

72 Loi organique portant organisation et fonctionnement de la CC, note 5, article 59.

73 Loi organique portant organisation et fonctionnement de la CC, note 5, article 63.

74 Loi organique portant organisation et fonctionnement de la CC, note 5, article 70.

75 Loi organique portant organisation et fonctionnement de la CC, note 5, article 83 alinéa 5. 
ou du Premier ministre. Il y a lieu de relever une fois de plus que pour ce dernier cas, toute personne intéressée peut saisir la Cour. Ceci s'explique par le fait que, en tant qu'autorité politique et par conséquent gérante de nos avoirs et de notre avenir, leur vie et richesse devraient concerner avec beaucoup d'intérêt toute la société, mieux tous les congolais qui, du reste, ont un droit fondamental au respect de la répartition équitable des richesses ${ }^{76}$. Pour ce cas de figure donc, les autorités fiscales, l'autorité de contrôle de l'exécutif et les ONGs de défense des droits humains sont mieux placés pour effectuer cette saisine sans sourcier.

En cas de vacances et dès la survenance de celle-ci, la Cour est saisie par le Gouvernement de la République réuni de toute urgence en Conseil des ministres ${ }^{77}$.

De cette analyse, il appert donc que les fonctions du PG peuvent être mieux exercées par d'autres structures et institutions faisant de ce dernier un apparent colosse dont les pieds se révèlent être de l'argile.

$\mathrm{Au}$ nom de la gestion saine et efficiente de l'Etat, supprimer cette institution serait un pas vers la bonne direction, lorsqu'on sait que la situation sociale de la population congolaise exige une attention soutenue du Gouvernement congolais.

\section{Une gestion efficiente de l'Etat}

L'article 13 de la loi organique portant organisation et fonctionnement de la $\mathrm{CC}$ dispose de manière très vague que le Procureur général est assisté d'un ou de plusieurs Premiers avocats généraux et d'un ou de plusieurs Avocats généraux sans en fixer le nombre. Il en résulte que ces magistrats pris au sein de l'ordre judiciaire ou administratif devront tout aussi faire l'objet d'un remplacement au sein de leur administration d'origine entrainant ainsi la hausse de l'enveloppe salariale d'un Etat au bord de la faillite ou sinon d'un Etat failli. Rajouter à cela que le PG fonctionne administrativement avec l'aide d'un secrétariat, dirigé par un Secrétaire général et fonctionnant avec l'aide d'un personnel permanent.

Il y a lieu de relever que le Procureur général jouit des avantages similaires à ceux des membres de la Cour. A ce titre, il a droit à une panoplie d'avantages allant de sa garde rapprochée, son véhicule de fonction et de résidence, son indemnité de logement, la sécurité de sa résidence, etc. ${ }^{78}$.

Or, il y a lieu de relever que la gestion efficiente de l'Etat résulte d'une conception anglaise de gestion de la chose publique qui veut à ce que celle-ci puisse être réalisée dans la même rigueur que celle qui préside le secteur d'activités économiques privées. Ainsi, à chaque fois que le Gouvernement aura la possibilité d'obtenir de bons résultats à faible coût, celui-ci devrait recourir à cette voie ${ }^{79}$.

76 Constitution de la RDC, note 18, article 58.

77 Loi organique portant organisation et fonctionnement de la CC, note 5, article 85.

78 Articles 13 à 21 de l'ordonnance $\mathrm{n}^{\circ} 16 / 070$ du 22 aout 2016 portant dispositions relatives au statut particulier des membres de la Cour constitutionnelle.

79 Darine BAKKOUR, un essai de définition du concept de gouvernance, www.lameta.univ-montp1.f r/Documents/ESS2013-05.pdf\&sa, 2013, p15, consulté le 22 juin 2018. 
Par conséquent, dans le but d'éviter donc tout bradage des biens de l'Etat, vu la modicité des pouvoirs accordés à cette institution et étant donné que l'expérience avérée en matière constitutionnelle des Procureurs près la Cc n'est pas de mise et surtout du fait que les tâches dévolues à ce dernier peuvent être éloquemment exercées par la société dans sa globalité, du fait de la large ouverture de la saisine de la Cour, nous proposons que cette dernière siège sans cet organe ou, à défaut, qu'elle siège avec le concours du PG près le Conseil d'Etat ou près la Cour de Cassation selon les cas. En effet, les contentieux administratif et constitutionnel quoique fondamentalement différents, se rapprochent en ce que les deux portent essentiellement sur les actes ${ }^{80}$. Ainsi, dans les matières autres que pénal, le PG près le Conseil d'Etat pourrait intervenir alors qu'en matière pénale, la Cour de cassation pourrait assurer cette charge, justifiant ainsi le recours dont il est question à l'article 18 de la loi organique portant organisation et fonctionnement de la Cc.

A la lecture des arguments ci-avant exposés, nous pensons que le fonctionnement du PG près la $\mathrm{CC}$, dans un pays dont les recettes ne sont pas à même de satisfaire aux desideratas de toute la population, est simplement budgétivore.

Dans le cas d'espèce, il a été démontré que cette institution budgétivore n'est rien d'autre qu'une guirlande sans fonction réelle que celle d'orner l'architecture institutionnelle. La maintenir reviendrait sans nul d'autre à brader l'argent de l'Etat au détriment de la population, violant ainsi le principe sacro-saint de redistribution équitable de richesse ${ }^{81}$ entre les différents membres de la population congolaise.

\section{Conclusion}

Dans un Etat où la création et la multiplication des institutions est récurrente, il n'est pas étonnant de retrouver des institutions d'un genre particulier et nouveau dans son exosquelette administratif. Le Parquet général près la Cour constitutionnelle s'inscrit dans cette veine.

Issu d'un souci d'innovation certes, le PG près la $\mathrm{CC}$ pèche en ce qu'il est doté des réels pouvoirs qu'il ne peut, sauf fiction, exercer véritablement du fait des contraintes liées à sa position de redevable vis-à-vis de l'exécutif et du fait que ses pouvoirs peuvent être exercés à mieux par d'autres organes et par tous les individus composant la société congolaise, bénéficiaires du droit de saisine de la Cour.

Ainsi, à la dernière marche de cette étude, nous proposons la suppression pure et simple de cet organe. A défaut, et pour satisfaire la tradition processuelle en matière d'administration de la justice, nous proposons que le pouvoir de cet organe soit conféré à son pendant

80 Gregory KALFLÈCHE, La notion de contentieux, une spécificité du droit public?, in l'identité du droit public, Travaux de l'IFR Mutation des normes juridiques, Sous dir. Xavier BIOY, Janvier 2011, pp. 159-174.

81 Article 58 de la Constitution de la RDC : « Tous les Congolais ont le droit de jouir des richesses nationales. L'Etat a le devoir de les redistribuer équitablement et de garantir le droit au développement. 
du Conseil d'Etat ou de la Cour de cassation selon qu'on se retrouvera respectivement devant un contentieux portant sur les actes ou devant un contentieux portant sur les personnes, comme en matière pénale précisément.

\section{Bibliographie}

André BUTEAU, «Les fonctions de poursuivant, de gardien de l'intérêt public, de représentant de l'État devant les tribunaux et de conseiller juridique exercées par le ministre de la Justice et Procureur général du Québec », in www.conferencedesjuristes.gouv.qc.ca/files/documents/21/84/lesfonct ionsdepoursuivantdegardiendelinteretpublic.pdf, consulté le 22/07/2018.

BALINGENE KAHOMBO, «L'originalité de la Cour constitutionnelle congolaise : son organisation et ses compétences », in www.hamann-legal.de, consulté le 22/07/2018.

Constitution de la République démocratique du Congo (RDC) telle que modifiée par l'article 1er de la Loi n 11/002 du 20 janvier 2011 portant révision de certains articles de la Constitution de la RDC, JORDC, 47ème Année, Numéro spécial du 18 février 2006.

Darine BAKKOUR, un essai de définition du concept de gouvernance, www.lameta.univ-montp1.fr/Do cuments/ESS2013-05.pdf\&sa, 2013, consulté le 22 juin 2018.

Félix VUNDUAWE te PEMAKO et Jean-Marie MBOKO D'JANDIMA, Droit constitutionnel du Congo, vol. II, Bruxelles, 2012.

Gregory KALFLĖCHE, La notion de contentieux, une spécificité du droit public?, in l'identité du droit public, Travaux de l'IFR Mutation des normes juridiques, Sous dir. Xavier BIOY, Janvier 2011.

Guy-Prosper DJUMA, Parquet général près la Cour constitutionnelle de la République démocratique du Congo, annuaire congolais de justice constitutionnelle, vol.1, 2016.

Jacques Van COMPERNOLLE, Le double degré de juridiction et les exigences du procès équitable, in "Le double degré de juridiction. Etude de droit comparé ", Sous dir., Jacques Van COMPERNOLLE, Achille SALETTI, Bruxelles, 2010.

Jean-François RENUCCI, «L'affaire Medvedyev devant la grande chambre : les « dits » et les « nondits » d'un arrêt important », 2010, in www.dalloz.fr.

Jean-François RENUCCI, «Un séisme judiciaire : pour la Cour européenne des droits de l'Homme, les magistrats du parquet ne sont pas une autorité judiciaire », 2009, in www.dalloz.fr.

Joseph KAZADI MPIANA, La révision constitutionnelle congolaise du 20 janvier 2011 : considérations critiques d'un citoyen (juriste), p. 12, in www.droitcongolais.info, consulté le 10 juin 2018.

Loi organique $\mathrm{n}^{\circ} 13 / 011$-B du 11 avril 2013 portant organisation, fonctionnement et compétences des juridictions de l'ordre judiciaire, JORDC, 54 ${ }^{\text {ème }}$ année, nº spécial du 11 avril 2013, 2013.

Loi organique $\mathrm{n}^{\circ} 13 / 026$ du 15 octobre 2013 portant organisation et fonctionnement de la Cour Constitutionnelle, JORDC, 54 $4^{\mathrm{ème}}$ année, numéro spécial du 18 Octobre 2013.

Marc-Antoine GRANGER, Le parquet, une autorité judiciaire indépendante?, in www.droitconstitution nel.org/congrèsNancy/comN6/grangerT6.pdf\&sa, consulté le 10 juin 2018.

Marcelin NGUELE ABADA, L'indépendance des juridictions constitutionnelles dans le constitutionnalisme des Etats francophones post-guerre froide : l'exemple du Conseil constitutionnel camerounais » in www.droitconstitutionnel.org, consulté le 09/06/2018. 
Maria Rosaria DONNARUMMA, Le statut juridictionnel du Chef de l'Etat et sa destitution, in www.no mos-leattualitaneldiritto.it/wp-content/uploads/2017/02/saggio-Donnarumma-Le-statut...-corretto.p df\&sa, consulté le 11 juin 2018

Pacte international relatif aux droits civils et politiques, adopté et ouvert à la signature, à la ratification et à l'adhésion par l'Assemblée générale dans sa résolution 2200 A (XXI) du 16 décembre 1966, entrée en vigueur le 23 mars 1976.

Philippe FOILLARD, Manuel de droit administratif, 14 ${ }^{\text {ème }}$ éd., 2009-2010.

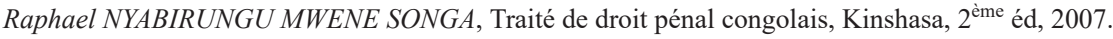

Stéphane BOLLE, « Vers une Cour constitutionnelle à la congolaise », in, http://www.la-constitution-en -afrique.org/ consulté le 07 juin 2018.

Yves SINTOMER, Le pouvoir au peuple. Jurys citoyens, tirage au sort et démocratie participative, Extraits, in www.etienne.chouard.free.fr, 2007, consulté le 18 juin 2018. 